\title{
HAADF STEM and EELS Investigations of Grain Boundaries in $\mathrm{SrTiO}_{3}$
}

\author{
K.J. Dudeck*, M. Couillard*, D.J.H. Cockayne** and G. Botton* \\ *Department of Materials Science and Engineering, McMaster University, Hamilton, Canada, L8S 4L7 \\ ** Department of Materials, University of Oxford, Oxford, United Kingdom, OX1 3PH
}

\begin{abstract}
Material structural and electronic properties are strongly influenced by the presence of grain boundaries, dislocations and defects. High resolution electron microscopy and spectroscopy are ideal tools for investigating the atomic structure of such defects as they can provide atomic scale information for comparison with models. Data for such comparison needs to be quantitative, with high accuracy and precision. Through use of a variety of complementary techniques, such as HAADF STEM, EELS,
\end{abstract} HRTEM and focal series reconstruction, a complete understanding of chemical and structural properties of these defects can be developed [1]. Grain boundaries provide extensive challenges for quantitative studies. Strain and misalignments of the two grains at the boundary can complicate STEM data, for example by reducing contrast or signal intensity, making analysis ambiguous [2,3,4]. In this work, aberration corrected HAADF STEM and STEM EELS have been combined to determine structural and chemical information about a variety of coincidence site lattice (CSL) and near-CSL grain boundaries in $\mathrm{SrTiO}_{3}$, including a near- $\Sigma 3(112)$ boundary. We have explored a variety of approaches for obtaining and analysing data from the near-CSL boundaries, in particular those associated with acquiring HAADF and EELS data simultaneously. Through the use of two-dimensional STEM EELS mapping, chemical information about the boundaries and defects has been obtained. Direct comparison of the HAADF data and EELS maps highlights both structural and chemical information and can yield conclusions that would not be easily obtained by either technique alone. Figures 1a presents a typical HAADF STEM image of the grain boundary. Periodic repeat units having a mirror symmetric configuration, as predicted by density functional theory [5], can be observed along the boundary, as highlighted in figure 1b. This is in agreement with a quantitative HRTEM imaging matching investigation of the same boundary [6]. Figure 2 presents preliminary data showing the simultaneously acquired HAADF and Ti spectrum image taken from an area similar to that indicated by the dashed rectangle on figure 1a. Statistical analysis of the spectrum image data is in progress.

\section{References:}

[1] W.M. Rainforth, Advances in Imaging and Electron Physics 132 (2004) 167.

[2] Z. Yu, D.A. Muller and J. Silcox, Ultramicroscopy 108 (2008) 494.

[3] L. Fitting, S. Thiel, A. Schmehl, J. Mannhart and D.A. Muller, Ultramicroscopy 106 (2006) 1053.

[4] V. Grillo, Ultramicroscopy 109 (2009) 1453.

[5] Benedek et al., Physical Review B 78 (2008) 064110.

[6] K.J. Dudeck and D.J.H. Cockayne, Journal of Physics: Conference Series accepted.

[7] Material courtesy of W. Sigle and M. Ruhle, and specimen preparation by Ute Salzberger (MPI Stuttgart) is acknowledged. 
(a)

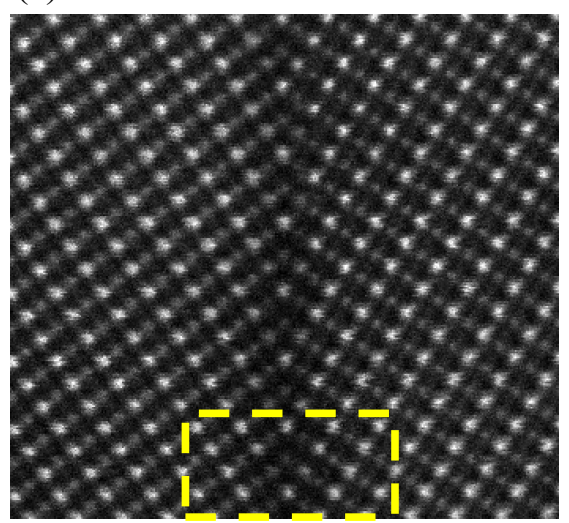

$1 \mathrm{~nm}$ (b)

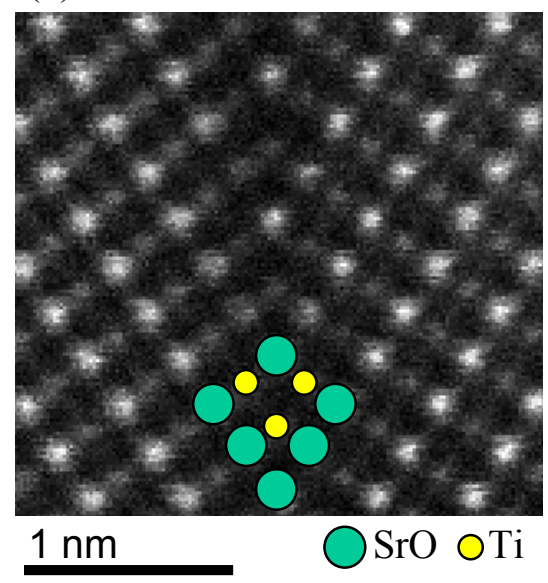

Figure 1: (a) Typical HAADF STEM image of the grain boundary; dashed rectangle indicates an area similar to that used for the two-dimensional spectrum imaging. (b) Enlarged view of the grain boundary with columns indicated.

(a)

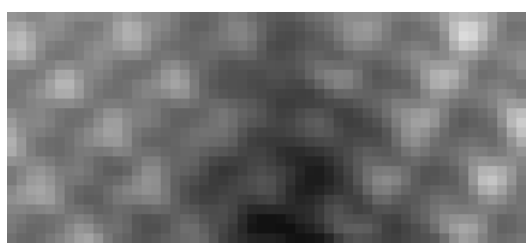

(b)

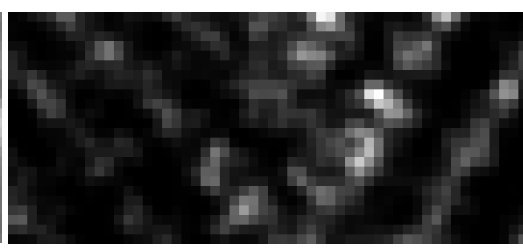

(c)

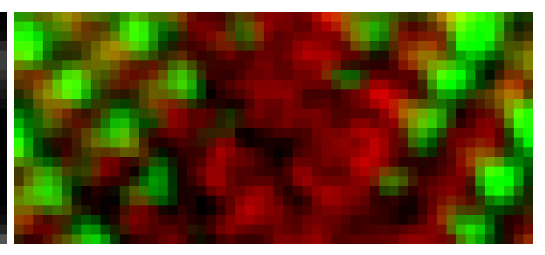

$1 \mathrm{~nm}$

Figure 2: (a) HAADF STEM image of the $\mathrm{SrTiO}_{3} \Sigma 3(112)[-110]$ grain boundary acquired simultaneously with EELS data. (b) Ti spectrum image corresponding to the same area as the HAADF image. (c) Ti spectrum image (red) overlayed onto the HAADF STEM image (green) (colour on CD proceedings). Note that a non-linear contrast scale has been employed when displaying the images. The area shown is similar to that indicated by the dashed rectangle in figure $1 \mathrm{a}$. 